\title{
A GEOMETRIC APPROACH TO THE HYPERBOLIC JØRGENSEN INEQUALITY
}

\author{
JANE GILMAN
}

Introduction. Jørgensen's inequality $[\mathbf{J}]$ gives a necessary condition for two elements of PSL $(2, \mathrm{C})$ to generate a nonelementary discrete group. If $A$ and $B$ are in $\operatorname{PSL}(2, \mathrm{C})$, the inequality says that

$$
\left|\operatorname{tr}^{2} A-4\right|+|\operatorname{tr}[A, B]-2| \geq 1
$$

where $\operatorname{tr}$ is the trace and [,] represents the commutator. This is one of the most useful and powerful tools available for determining nondiscreteness. The precise geometric meaning of this inequality has been unclear.

Here we first give an equivalent but more geometric formulation of the inequality (see (II)) in the case of hyperbolic elements in $\operatorname{PSL}(2, \mathbf{R})$. Next we outline a proof of the inequality for the case of purely hyperbolic subgroups of $\operatorname{PSL}(2, \mathbf{R})$ which shows that under these circumstances an even stronger inequality is satisfied (see (III)). For purely hyperbolic discrete groups (I) is trivially satisfied for all but the finite number of conjugacy classes of elements (or their inverses) with multiplier between 1 and $(3+\sqrt{5}) / 2$, whereas the stronger inequality is not.

Groups of Möbius transformations in space are an increasingly important area of study. A Jørgensen type inequality would be significant there, and it is hoped that this formulation of Jørgensen's inequality might point the way toward the proper formulation in space.

I wish to thank Professor B. Maskit for pointing out to me the significance of this formulation, and Professor D. Gallo, who was the first to ask whether the inequalities in [G] implied Jørgensen's inequality.

The inequalities. Let $A$ and $B$ be hyperbolic matrices with multipliers $R$ and $K$ respectively. If $v_{A}, w_{A}$ and $v_{B}, w_{B}$ are the repelling and attracting fixed points of $A$ and $B$ respectively, let $C$ be the cross ratio

$$
\left(\left(v_{A}-v_{B}\right)\left(w_{A}-w_{B}\right)\right) /\left(\left(v_{A}-w_{B}\right)\left(w_{A}-v_{B}\right)\right) .
$$

Note that $C, R$, and $K$ are conjugation invariant.

Let $f(x)$ be the function $f(x)=x /(x-1)^{2}$ and note that $f(x)=f(1 / x)$. Compute that $\operatorname{tr}[A, B]=2+f(C) /(f(R) f(K))$ (see [G]). Rewrite (I) as

$$
\frac{1}{f(R)}+\frac{|f(C)|}{f(R) f(K)} \geq 1 \text {. }
$$

Received by the editors August 1, 1986.

1980 Mathematics Subject Classification (1985 Revision). Primary 30F35, 20H10.

Research supported in part by NSF Grant No. 8120790 . 
THEOREM. If $A$ and $B$ generate a nonelementary purely hyperbolic subgroup of $\operatorname{PSL}(2, \mathbf{R})$, then

$$
\frac{|f(C)|}{f(R) f(K)}>4 .
$$

OUTLINE OF PROOF. In $[\mathbf{G}]$ we show (using a proof that is independent of Jørgensen's inequality) that a nonelementary subgroup of $\operatorname{PSL}(2, \mathbf{R})$ is discrete and torsion free if and only if for each pair of hyperbolics which generate a nonelementary subgroup one of three inequalities holds. One then shows that when combined with results from $[\mathbf{R}]$ each of the three inequalities implies (III).

Let $T_{A}$ and $T_{B}$ be the translation length of $A$ and $B$ respectively. There is a relation between $C$ and the distance between the axes of $A$ and $B$ when they are disjoint and between $C$ and their angle of intersection when they are not disjoint. Using the relation and (III) one can obtain

COROLLARY. If $A$ and $B$ generate a nonelementary purely hyperbolic discrete subgroup of $\mathrm{PSL}(\mathbf{2}, \mathbf{R})$, then

(IVa) if the axes of $A$ and $B$ are disjoint and $D$ is the length of the common perpendicular to the axes,

$$
\left(\sinh ^{2} D\right)\left(\sinh ^{2}\left(T_{A} / 2\right)\right)\left(\sinh ^{2}\left(T_{B} / 2\right)\right)>1,
$$

and

(IVb) if the axes of $A$ and $B$ meet at angle $\theta$,

$$
\left(\sin ^{2} \theta\right)\left(\sinh ^{2}\left(T_{A} / 2\right)\right)\left(\sinh ^{2}\left(T_{B} / 2\right)\right)>1 .
$$

\section{REFERENCES}

[G] J. Gilman, Inequalities and discrete subgroups of $\operatorname{PSL}(2, \mathbf{R})$, preprint.

[J] T. Jørgensen, On discrete groups of Möbius transformations, Amer. J. Math. 98 (1976), 739-749.

A note on subgroups of SL(2, C), Quart. J. Math. Oxford Ser. II 28 (1977), 209-212.

[R] G. Rosenberger, Real two dimensional representations of two-generator free groups, Math Z. 127 (1972), 95-104.

All generating pairs of all two-generator Fuchsian groups, Arch. Math. 46 (1986), 198-204. 94720

Mathematical SCIENCES RESearch Institute, Berkeley, California

Department of Mathematics, Rutgers University, Newark, NeW JerSEY 07102 